\title{
Las bodas de plata de una lluvia de oro. Veinticinco años de teología católica latina en Estados Unidos
}

\author{
Kenneth G. Davis, O.F.M. \\ Oblate School of Theology, \\ San Antonio, Texas.
}

En 1968, Gustavo Gutiérrez pronunció un famoso discurso en Chimbote, Perú, que sirvió de base a su libro La teología de la liberación, el cual, a su vez, inició el movimiento del mismo nombre. Los exponentes de esta teología son ampliamente conocidos y su influjo se nota desde las junglas de Chiapas hasta la catedral de Canterbury.

Para los latinos y latinas que viven en Estados Unidos, sin embargo, más importante aún, aunque en alguna forma menos reconocido, ha sido el trabajo de Virgilio Elizondo. El movimiento de teología católica contemporánea de los latinos y latinas de Estados Unidos, que él inicio, se acerca a sus bodas de plata, I y eso es lo que queremos celebrar y analizar en este artículo. Este movimiento nos parece especialmente relevante porque funge como puente entre diversas teologías, aunque, por falıa de espacio, no podamos entrar en este arlículo en el análisis de la teología hispana protestante de Estados Unidos, tema importante y relevante.2

1. Piensan algunos que el primer trabajo teológico de Virgilio Elizondo fue publicado en 1968, pero el historiador Timolhy Matovina ha demostrado que su publicación en Catequesis latinoamericana apareció realmente en 1972, con lo cual nos acercamos al XXV aniversario. Elizondo reconoce públicamente su deuda con Gutiértez, y por esta razón menciono a Gustavo como antecesor inmediato de la teologia católica de los hispanos en Estados Unidos. Véase Eduardo Femández, S. J., "Towards a U. S Hispanic Theology: A Study of a Current Bibliography". Tesis de licenciatura, no publicada, de la Universidad Pontificia Gregoriana, 1992.

2. Los límites de este artículo no permiten el análisis de las diversas teologías hispanas protestantes, igualmente variadas y ricas, Jaguna que procuraré llenar en futuras publicaciones. Para un estudio de los principales autores protestantes hispanos véase Justo 
Digamos, por último, para terminar esta introducción, que nos concentramos, y reducimos, en nuestro análisis al movimiento teológico latino de la parte continental de Estados Unidos de América. ${ }^{3}$

\section{La primera década: $1968-1978$}

Bajo el influjo de Gustavo Gutiérrez, quien insiste en que las teologías locales tienen que escuchar a los indígenas, a los oprimidos y a otras voces silenciada en Estados Unidos, los latinos tuvieron que precisar, ante todo, quién era el sujeto de su teología, es decir, quiénes eran ellos. Esto ya lo habjan hecho los europeo-americanos que conquistaron sus tieras, y lo continúan haciendo. Así, el gobernador actual y los dos senadores de Califomia parecen pensar que los latinos son un grupo sospechoso, que sólo empobrece las finanzas del gobierno. Los dominicanos y los colombianos son normalmente tenidos por sospechosos y traficantes de droga. Los portorriqueños son popularmente considerados como extranjeros. 4 Incluso los ciudadanos de sus tierras de origen los discriminan. Los consideran "agringados", que hablan el "pocho", que no son de pura casta. La conclusión es que el latino que ha nacido en Estados Unidos sufre un doble rechazo.

Pues bien, tal como sucede con olras minorías concientizadas, Elizondo hace uso de ese rechazo para definir el sujeto de la teología: el mestizo. El mestizaje está relacionado con lo biológico, lo cultural y lo religioso, y con la mezcla de personas, que, en el caso de pueblos conquistados, es una mezcla violenta y rapaz.5 En este contexto, Elizondo presenta a Cristo como paradigma de esta realidad. Así como Cristo fue rechazado tanto por la élite de los israelitas de su tiempo, como por los conquistadores romanos, de igual forma, los mestizos contemporáneos son marginados tanto por los antepasados "puros" de su propia

González, Voces: Voices from the Hispanic Churh, Nashville, 1992. Lara Medina intenta establecer algún tipo de correlación entre las teologlas protestantes y católicas de Estados Unidos en "Broadening the Discourse at the Theological Table: An Overview of Latino Theology 1968-1993", Latino Studies Joumal 5 (3) septiembre 1994, pp. 10-36.

3. No está incluida la teología de la isla de Puerto Rico. En futuros trabajos espero analizar los escritos del difundo obispo jesuita Antonio Parrilla-Banilla. Para teología de Puerto Rico en Estados Unidos, pueden consultarse los escritos de Anthony Stevens-Arroyo. especialmente Prophets Denied Honor, Nueva York, 1980.

4. Nicolás Kanellos (ed.), The Hispanic American Almanac, Detroit, 1993.

5. Virgilio Elizondo, "Mestizaje as a Locus of Theological Reflection", en Allan Figueroa Deck. S. J. (ed.). Frontiers of Hispanic Theology in the United Stares, Nueva York, 1992. Otros latinos no siempre se identifican con el termino mestizo (aunque si aceptan el concepto). Algunos portorriqueños, por ejemplo, prefieren el término criollo. Para un análisis del têrmino véase Robert S. Goizuela, “ ¿La raza cósmica? The Vision of José Vasconcelos", Journal of hispanic-Latino Theology 1(2) febrero 1994, pp. 5-27. 


\section{LAS bodas de PLATA dE UNA LLUVia de gRo}

tierra de origen, como por los conquistadores extranjeros. 6 AsI como Jesús fue un judío marginado que sufrí un doble rechazo y opresión, pues confrontó en su propia cultura a los judfos que se autojustificaban y a los opresores extranjeros violentos, asi el mestizo sufre este doble rechazo y puede tener, por ello, una comprensión privilegiada de Jesús y del mestizaje.

Desde esta lúcida percepción de la realidad del latino en Estados Unidos se puede volver al sujeto de la teologla y hacer así una teología propia, en lugar de aceptar aquellas que integran, acríticamente, ambos rechazos. Es cierto que Elizondo está en deuda con Gutiérrez y que se inspira en él, pero tanto Elizondo como la teologla en general necesitaban dirigir la mirada a la experiencia única de su propio pueblo mestizo con el objeto de encontrar su locus theologicus. Y es que ni la teologia de Europa ni la de America Latina pueden enfocar adecuadamente la experiencia especffica del latino como sobreviviente de la doble opresión mencionada, tanto cultural como sociopolítica y económica. 7 Como méxico-americano que es, Elizondo escogío a la Virgen mestiza, Nuestra Señora de Guadalupe, para simbolizar el mestizaje. Otros latinos siguieron su ejemplo, utilizando sus propias expresiones religiosas populares, porque las vieron como "una reserva de valores de libertad, un dar fuerza a las víctimas, un guardián de la cultura hispana, un medio de evangelización, y también un balance a ciertos valores dominantes en la Norteamérica postmoderna". 8

En esa época también comenzaron a ser escuchadas otras voces hispanas. Allan Figueroa Deck, S. J. comenz6 a publicar sus escritos en 1974, y desde entonces ha centrado la mayor parte de su trabajo en la religiosidad populer con la finalidad especifica de evangelizar la cultura moderna y secular en la que viven los hispanos en Estados Unidos. De esta forma ha proseguido la tendencia

6. Virgilio Elizondo, Galilean Joumey: the Mexican-American Promise, Nueva York, 1983. Este trabajo es una versión popular de su disertación Mestissage, Violence Culturelle. Annonce de l'evangile, presentada en el Instituto Católico de París en 1978. Esta obra puede servir para delitar el primer período de la evolución de esta teologfa latina, tan claramente dominado por Elizondo.

7. La mayoría de los teólogos latinos hacen uso de embes tradiciones. En verdad muchos de los téblogos latinos en Estados Únidos recibieron su doctorado o tuvieron su experiencia educativa en uno de esos continentes. Sin embargo, ellos no están ni en el centro de poder económico y político, como los curopeos, ni en la casa ancestral de sus culturas, como los latinoamericanos. Por ello, aunque usan las tradiciones intelectuales de ambas regiones, insisten tambien en que, como su experlencia es única, su teologfa debe ser distinta. Véase Sixto Gercla, "Sources and Loci of Hispanic theology", Journal of Hispanic-Latino Theology I(I) noviembre 1993, pp. 22-43.

8. Arturo Bafiuelas, "U.S. Hispanic Theology", Misslology XX (2) abrll 1992, pp. 275300. Para une bibliografla mAs complete véase su artículo en Apunies $11(4)$ invierno 1991. 
praxológica del trabajo de Elizondo, probablemente con un uso más sofísticado de las ciencias sociales en el análisis de la realidad. Deck y otros teólogos hispanos de Estados Unidos vieron con buenos ojos la religiosidad popular aun anies - y con mayor pasión - que sus colegas latinoamericanos. Recordemos que el misıno Guslavo Guliérrez todavía ignoraba el tema en su segunda edición de La teología de la liberación.

Ora importante característica de esta primera década es el papel crucial de la mujer hispana. En 1975, varias mujeres dieron nuevos aportes, que fueron publicados. 9 Aunque todavía eran una minoría entre los teólogos, fueron pioneras de la extremadamente importante experiencia de cooperación teológica entre los latinos y latinas, llaınada también teología de conjunto. Las semillas de este experimento teológico fueron sembradas en diferentes lugares.

En 1971. las religiosas hispanas iniciaron la organización LAS HERMANAS, que, posteriormente, a diferencia del grupo de sacerdotes (PADRES), fomentó la colaboración entre las mujeres hispanas de diferentes procedencias étnicas. $10 \mathrm{Al}$ año siguiente participaron en la fundación del Centro Cultural México-Americano (MACC) en San Antonio, Texas. En la primera junta de directores, en la que había rcligiosas, laicos, clérigos y obispos," " había ya cuatro mujeres.

Pero tal vez la scmilla más importante, que eventualmente animó a la cooperación en todos los niveles de la Iglesia hispana de Estados Unidos, fue el proceso del encuentro nacional en el que las mujeres jugaron un papel importantc. En esta primera década se realizaron dos reuniones nacionales de liderazgo hispano, que culminaron en el tercer encuentro de 1985, tras un proceso de dos años de consulta nacional. Tanto este ministerio de cooperación como la cuidadosa atención a la experiencia del pueblo han influido en la reflexión teológica hispana. 12 Los dos primerosencuentros promovieron la creación de centros ministeriales regionales, algunos de los cuales comenzaron a publicar trabajos

9. Quizás la más prominente fue Marina Herrera, pero no estuvo sola. Véase Marfa Consuelo Pacheco, Liberty and Justice for All: Nationhood a View from the Mexican American Community. San Antonio, 1975, así como los publicaciones de Macc de Angela Erevia.

10. Arroyo (ed.) Prophets Denied Honor, p. 136.

11. Véase "LAS HERMANAS" de Timothy Malovina y Yolanda Tarango, en Philip Lampe (ed.). Hispanics in the Church: Up from the Cellar, San Francisco, 1994. Véase también, Kenneth Davis, "CORHIM: Hispanic Seminars", Review for Religious, noviembre-diciembre, 1991, pp. $881-887$.

12. Para el análisis de cómo la experiencia, particularmente de las latinas, es utilizada como fucnte vital de la teología, puede leerse tres artículos escrilos desde diferentes perspectivas, aunque complementarias, de María Pilar Aquino, Ada Marfa lsasi-Díaz y Jeanelte Rodríguez en la primera edición del Joumal of Hispanic/Latino Theology. 
teológicos. Otros, como el Instituto de Pastoral del Sudest5.desanolkanon, eventualmente, escuelas de reflexión teológica. ${ }^{13}$

\section{La segunda década: $1979-1989$}

Las semillas brotaron en tierra fértil. El número de personas que se identificó con este modo de hacer teología comenzó a crecer y a extenderse dramáticamente en la siguiente década. La tendencia praxológica continuó con importantes escritos de ministros pastoralistas, 14 incluyendo una sorprendente cantidad de laicos. Aun en comparación con los protestantes hispanos de Estados Unidos, son más numerorosos los escritores católicos laicos. 15 Adernás, mientras los poravoces y los escritores de esta leología se extendían y diversificaban, el número de personas que vivía la experiencia, que reflexionaba sobre ella y la compartía con los teóricos, crecía aun más dramáticamente a través del proceso del tercer encuentro, que culminó con la ya mencionada asamblea nacional de 1985.

Fueron los mismos obispos de Estados Unidos los que convocaron al tercer encuentro, y durante todo el proceso escucharon las experiencias de más de cien mil hispanos. Y no sólo las escucharon cuidadosamente, sino que también, conscientes de esa realidad, hicieron su propio análisis. Como resultado de todo ello se elaboró un plan de acción llamado el Plan pastoral nacional del ministerio hispano. Los obispos apoyaron un método de reflexión praxológico liberacionista, basado en una eclesiología de cooperación, que es el método y la eclesiología que utilizan la mayoría de los teólogos hispanos. Por ello, no es una coincidencia casual que esta pastoral de conjunto dé lugar a una teología de conjunto.

Varios leólogos participaron en los encuentros y muchos puntos específicos de interés del Plan del Ministerio Hispano (por ejemplo las mujeres y el pobre) son temas constantes en sus escritos. De igual forma, así como el Plan insiste en la formación de líderes, muchos de los teólogos son también profesores o formadores. Esta interrelación entre prioridades pastorales y preocupaciones teológicas está arraigada en el acompañamiento del pueblo, que los teólogos llevan a cabo conscientemente, y en las melas de su teología: articular una comprensión de Dios que ayude a los latinos y latinas a liberarse de todo lo que los oprime.

Esa pudiera ser también una de las razones por qué, a diferencia de algunos de sus colegas latinoamericanos, los teólogos de Estados Unidos no han tenido

13. Soledad Galerón, Rosa María Icaza, Rosenda Urrabazo (eds.), Prophet Vision: Pastoral Reflections on the National Pastoral Plan for Hispanic Ministry, San Antonio, 1992.

14. Véase los trabajos de Arturo Pérez tales como Popular Catholicism, Washington, 1988.

15. Más de media docena de laicos empezó a publicar en esa década, entre ellos David T. Abalos, Latinos in the Unired State: The Sacred and the Political, Notre Dame,

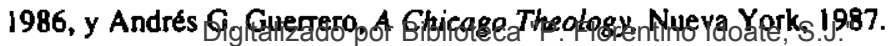

Universidad Centroamericana José Simeón Cañas 
mayores problemas con su jerarqula eclesiásica. Otra razón puede ser el hecho de que muchos de los obispos han participado en el diálogo teológico, como, por ejemplo, Roberto $O$. González y Ricardo Ramírez. Cualquiera que sea la razón, se nota cierto apoyo de los obispos a la metodología y a las fuentes de esta teologia, aunque no siempre a las conclusiones a las que ésta llega.

Como consecuencia de todo ello, se ha ampliado el cf́rculo de té́logos, el cual llega a incluir literalmente a miles de personas, aunque todavia son muy pocos los que publican o los exponentes formales de esa teología. En todo caso, el pueblo, los obispos y los télogos generalmente están de acuerdo en los métodos y las fuentes de la teología: la realidad del pueblo, especificamente su opresión (véase la opción del Plan por los pobres y marginados), y la religiosidad popular (a la cual el Plan llama mística). 16

En esta segunda década, a medida que Virgilio Elizondo comenzó a ser reconocido internacionalmente, más y más latinos y latinas de Estados Unidos se apoyaron en su teologla, y muchos de estos esfuerzos dieron su fruto. Por ejemplo, comenzaron a articularse mejor las experiencias de las mujeres. Ada Marla Isasi-Dlaz publicó un articulo pionero "'Apuntes' for a Hispanic Women's Theology of Liberation", 17 al que siguió su trabajo en colaboración con Yolanda Tarango, Hispanic Women: Prophetic Voice in the Church.18 Muchas otras mujeres comenzaron a obtener títulos avanzados en teología o en estudios relacionados con ésta. Marla Pilar Aquino comenzo a publicar, prolfficamente, en 1980, y Ana Marla Pineda, en 1988. Rosa Marla Icaza y Marla de la Cruz Aymes, aunque escriben sobre liturgia y catequesis respectivamente, han influido también sobre pensadores y teólogos hispanos. 19

Al mismo tiempo que Virgilio Elizondo hablaba ante la Sociedad Teologica

16. El análisis social usado en los encuentros ha sido criticado por ser demaslado simple. Esto ha ido mejorando, de alguna manera, en los últimos cinco años. Quisiera hacer notar, sin embargo, que estamos seffalando tendencias que dan esperanza més que realidades consumadas. Asf, no se puede decir que el trabajo de las mujeres y de los pobres, como agentes de su propio destino, sea un hecho ye logrado, pero hay esperanzas de ello.

17. Este articulo ha sido reimpreso, junto a otros trabajos importantes anteriores, en Justo L. González (ed.), Voces. Esta es una colección de algunos artículos de la publicación protestante hispano Apuntes: Reflexiones Teologicas desde el Morgen Hispano, que se inició más de una década antes que The Journal of Hispanic/Latino Theology.

18. Publicado en San Francisco, en 1988. Ambas mujeres son miembros desde hace mucho tiempo de LAS HERMANAS. Véase también Marfa Pilar, Our Cry for Life, Nueva York, 1993, y Ada Maria Isasi-Dlaz, En la Lucha: An Hispanic Women's Liberation Theology, Nueva York, 1993.

19. Icaza ayudo en la edición billngle de la publicación de Sheed y Ward Vision profética en 1992. 
Católica de América (CTSA) en 1975 y 1981, al final de la segunda década Sixto J. Garcia y Orlando Espín hacian un importante aporte precisamente sobre religiosidad popular. Ambos sostienen que ésta debe ser considerada como parte privilegiada del sensus fidelium, y desde ahl elaboran una teologia de la gracia y la providencia. ${ }^{20}$ Mientras que los teólogos chicanos han tratado, casi exclusivamente, de nuestra Señora de Guadalupe (que puede convertirse en símbolo religioso universal hispano), éstos y otros teólogos ahondan en las expresiones religiosas populares de los portorriqueños, cubanos, centro y sudamericanos y sus descendientes en Estados Unidos.21 En conclusión se puede decir que surge un consenso de que la religiosidad popular es (1) un recurso auténtico de catolicidad hispana, (2) un guardián de esa cultura en la fase de conquista, (3) un componente poético y espiritual necesario para cualquier teología, (4) un instrumento de liberación y esperanza, y (5) un medio para la evangelización de toda la Iglesia.

Algunos se han preguntado por qué tanta preocupación por lo intra-eclesial. ¿Por qué no poner más atención explícita al análisis social de la realidad, ya sea en América Latina o en los movimientos de derechos civiles de Estados Unidos? De hecho, los chicanos George Bach y Rita y Antonio Soto han criticado a la Iglesia por este fallo, mientras que Joseph Fitzpatrick S. J. ha fomentado el análisis sociológico de los portorriqueños. Por ello, aunque la crítica sea válida, no se puede universalizar, $y$, además, la situación, afortunadamente, está cambiando.22

Es cierto, pues, que unos teólogos enfatizaron la opresión cultural y otros la opresión socio-económica, pero ninguno de ellos vio ambos aspectos como separados o mutuamente excluyentes. De hecho afirman claramente que la liberación de una opresión ayudará a la liberación de la otra.23 Esta necesidad de

20. Veanse sus contribuciones en los Proceedings of the Catholic Theological Society of America, 1987-1989.

21. Véase los escritos de Antonio M. Stevens Arroyo o de Jaime Vidal, "Popular Religion in the Lands of Origin of New York's Hispanic Population", Hispanics of New York: Religious, Cultural and Social Experiences, Volume II, Nueva York, 1982.

22. Vease Kenneth Davis, "Church Must Empower Chicano", East Bay Monitor, noviembre 1990 , p. 7. Una de las razones de que en los inicios hubiera falta de diálogo entre los télólogos y los cientificos sociales latinos es que los primeros sociólogos que estudiaron la religión hispana eran también sacerdotes y, de alguna forma, teologos, pero la mayorfa dejó el sacerdocio. Por ello, durante algún tiempo nadie unificó las diferentes disciplinas.

23. Véase el capttulo segundo de mi libro Primero Dios: Alcoholics Anonymous in the Trearment of the Hispanic, Londres y Toronto, 1994. En general, los te6logos que nacieron fuera del país parecen enfatizar la opresión socioeconómica, mientras que los nacidos en Estados Unidos parecen estar más preocupados con la opresión cultural. 
enfrentar la doble opresión - experiencia típica del mestizo- es precisamente lo que hace necesaria la elaboración de una teología hispana de Estados Uni$\operatorname{dos}^{24}$ y lo que le dará identidad.

Así como con la disertación de Elizondo en 1978 se cerró la primera década de esta teología, caracterizada por el trabajo de unos pocos valientes luchadores - aunque a menudo en aislamiento-, así la fundación de la Academia de Teólogos Hispanos Católicos de Estados Unidos (ACHTUS) en 1989 dio inicio a la segunda década, en la que se advierle ya una mayor colaboración entre todos. Las siguientes palabras del boletín ACHTUS explican bien las fuentes, los métodos y las metas de esta teología:

Existen dos áreas que vienen a la mente y ejemplifican las oportunidades para nuestra Academia. La primera es fundamentalmente cultural y antropológica: la importancia central de la religiosidad popular como expresión de la vida de fe de nuestro pueblo. La segunda es socio-económica y socio-política: la pobreza masiva y la falta de poder, característica de nuestras comunidades. 25

A partir de esta experiencia pastoral y de esta praxis, hay que hacer una reflexión teológica... una teologia de conjunto... tocando temas como los de la justicia y la liberación de las estructuras opresivas. 26

Nuestra primera responsabilidad y nuestra obligación subsiguiente es hacia nuestro pueblo... El juntarnos no es solamente para compartir la experiencia común de un grupo privilegiado [sino para compartir] la experiencia común de la gran mayoría de nuestro pueblo en sus esfuerzos diarios para comprender la acción liberadora de Dios en sus vidas... el papel de nuestra teología es dar voz a lo que el pueblo dice y que no está siendo escuchado.27

\section{Una cosecha generosa: de 1990 hasta la fecha}

Los últimos años han visto, en primer lugar, el fenómeno acelerado de publicaciones teológicas enfocadas hacia el año 1992, el quinto centenario, como lo ha documentado el artículo anual del mes de marzo de Review for Religions,

24. También Virgilio Elizondo ha ampliado, gradualmente, su comprensión del mestizaje. Véase The Future is Mestizo: Life Where Culture Meets, Bloomington, 1988. Del mismo autor, "Hispanic Theology and Popular Piety: For Interreligious Encounter of a New Ecumenism", The Catholic Theological Sociery of America Proceeding (48) 193. pp. J-14.

25. Allan F. Deck, S. J., "Presidential Adress at the Firts Regular Meeting" ACHTUS, 1989 (texto no publicado).

26. ACHTUS Newsletter 1 (1) invierno, 1989-1990.

27. ACHTUS Newsletter 3(1) primavera, 1992. 
titulado "U.S. Hispanic Catholics: Trends and Recent Works . Esta explosion en las publicaciones ha sido acompañada por la tendencia a una mayor accptación de los teólogos latinos en las facultades de teología y en proyectos de investigación. En segundo lugar, se está dando una creciente especialización y maduración de la teología, que se expresa en un comienzo de diálogo crítico entre los teólogos. Por último, ha aumentado el diálogo entre esta teología latina y otras disciplinas.

Por lo que loca a las publicaciones, mencionemos algunos cjemplos importantes. En estos años, varios periódicos han dedicado números enteros a la realidad hispana.28 También se han publicado antologías 29 y un nuevo volumen sobre la Escritura. 30 En el año 1995 se volvieron a publicar nuevas antologías, una de ellas editada por Ana María Pineda con Robert J. Schreiter, mientras que Ada María Isasi-Díaz y Fernando F. Segovia están trabajando en otra. Jeanette Rodríguez publico Our Lady of Guadolupe: Faith and Empowernent Among Mexican American Women y Ana María Díaz Stevens ha publicado recientementc Oxcart Catholicism, que trata de las expresiones religiosas de los portorriqueños en Nueva York. "I La editorial de la Universidad de Nolre Dame publicó un estudio en tres volúmenes sobre la historia de la Iglesia hispano católica de Estados Unidos y Timolhy Malovina publicó un estudio histórico de casos sobre la religión y la etnicidad de los méxico-americanos titulado Tejano Religion and Ethnicity: San Antonio, 182/-1860 (Universidad de Texas, 1995). La traducción al inglés del libro de María Pilar Aquino Nuestro clamor por la vida fue publicada por la Editorial Orbis, y dicha editorial publicó también, en 1995, libros de Roberto Goizueta, Arturo Bañuelas y Alejandro García-Rivera. En noviembre de 1993 comenz6 a publicarse The Journal of Hispanic-Latino Theology.32The Living Light dedicó la edición de invierno de 1994 al cincuenta aniversario de la Secrelaría de Asuntos Hispanos. Ese mismo año, el periódico Sociedad y cristianismo, de Ecuador, dedicó la edición número $31 / 2$ a las iglesias lalinas de Estados Unidos. En 1994 y 1995, Religioni e Società de Florencia publicó una serie

28. Edición de invierno primavera, 1990 de U.S. Catholic Historian, y la edición de diciembre de 1991 de Listening: Journal of religion and Culture. También. Missiology op. Cit. y la edición de diciembre de 199| del New Theology Review.

29. Roberto S. Goinzueta (ed.), We are a People: Iniriatives in Hispanic American Theology, Minneapolis, 1992. Eugene F. Hemrick (ed.) Strangers and Aliens No Linger: Part One the Hispanic Presence in the Church in the United States, Washington, D. C. 1993.

30. C. Gilbert Romero, Hispanic Devotional Piety: Tracing the Biblical Roots, Nueva York, 1991.

31. Notre Dame, 1993. El libro de J. Rodríguez fue publicado en 1994 por la Universidad de Texas.

32. Mayor información sobre esta nueva revista se puede obtener en The Liturgical Press,

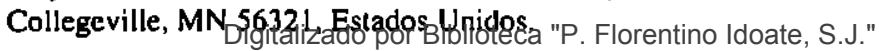

Universidad Centroamericana José Simeón Cañas 
de artículos sobre la experiencia religiosa de los hispanos. Por último, la reunión de 1993 de la Sociedad Teológica Católica de América dedicó una buena parte de su tiempo (un veinte por ciento) a ponentes latinos, cosa que nunca antes habla ocurrido. ${ }^{33}$ Los talleres sobre la problemática de los latinos y latinas son ya habituales en las reuniones de la Sociedad Teológica Católica de América.

Los latinos y latinas están entrando - lentamente- a formar parte de las facultades teológicas. Sólo en los últimos cinco años, las universidades de San Diego, Loyola, Seattle, Loyola Marymount, Notre Dame, Drew, St. Peter College, las escuelas de teologla de los jesuitas (Berkeley), de los franciscanos, de los oblatos, el Catholic Theological Union, los seminarios Sacred Heart y Saint Thomas, la universidad pontificia de los josefinos, entre otras instituciones teologicas, han agregado profesores hispanos a sus departamentos de teología y pastoral.

Por lo que toca al diálogo intrateológico, los teólogos han madurado en el enfoque de sus intereses particulares y han establecido un diálogo crítico entre ellos. Orlando Espín está desarrollando una teología sobre la gracia, Sixto Garcla sobre la trinidad y Roberto Goizueta, una teologla fundamental. Alan Deck continúa trabajando sobre la evangelización de las culturas y cada vez más té́logas latinas, como Gloria Inés Loya, están elaborando ricas y variadas teologías feministas. Fernando Segovia, Gilbert Romero, Jean Pierre-Ruiz y otros están publicando sobre Escritura. Segovia, Deck y Arturo Bañuelas han publicado ya diversas evaluaciones de este proyecto teológico y existe un diálogo continuo entre ellos, no sólo en reuniones formales, sino a través de recensiones de libros y de proyectos en colaboracion. ${ }^{34}$ Como conclusión podemos decir que, al tomar en cuenta las críticas de feministas y de los latinos no chicanos, el propio pensamiento de Virgilio Elizondo se ha desarrollado y profundizado. 35

Por último, también va en aumento la interdisciplinaridad de esta especifica teología de la liberación. Psicólogos, sociólogos, teólogos y pastores, todos co-

33. En esa reunión también apareció con claridad otra diferencia entre los teologos hispanos y los anglosajones. Los latinos son más jovvenes.

34. Ya existe una tercera generación de estudiosos que están evaluando, en tesis y disertaciones, esta rica herencia. Véase, por ejemplo, Claudia Burgaleta, "Can Syncretic Christianity Save? A Proposal for a Christian Recovery of the Syncretic Elements in Latin American Popular Religiosity Based on Rahner's Concept of Anonymous Christianity". Tesis M. S. no publicada de la Escuela Jesuita de Teologfa, Berkeley, 1992. Véase tambien Eduardo Fernandez, "Reading the Bible in sparish: U.S. Catholic Hispanic Theologians Contributions to Systematic Theology", Apunses 14 (3) otoño, 1994, pp. 86-90.

35. En conversación personal del 30 de septiembre de 1993, Virgilio Elizondo me decla que también ha sido influenciado por los contactos pastorales con la gente ordinaria y por los contactos ecuménicos, a los que ha tenido acceso como miembro de la Asociación Ecumbnica de los Teologos del Tercer Mundo. 
operaron en la producción de Stranger and Aliens No Longer. Part'One, The Hispanic Presence in the Church of the United States. 36 Anthony Stevens Arroyo, presidente del Programa para el Análisis de la Religión entre Latinos (PARAL), está patrocinando con éxito una cooperación interdisciplinar, que comenzo con una conferencia en la Universidad de Princeton, en 1993, y está editando una serie de libros basados, en parte, en este proceso. Gilbert Cadena esta preparando la publicación de A Broken Covenant: Chicano History and the Catholic Church, y Otto Maduro continúa su estudio interdisciplinar sobre la religión entre los latinos. También Philip Lampe ha editado un estudio sociológico sobre el catolicismo hispano de Estados Unidos. ${ }^{37}$

Para esta teología es fundamental el diálogo entre expertos y teólogos académicos con la experiencia del pueblo. Por ello, es muy significativa la celebración del primer congreso hispano (propuesto para cada tres años) y patrocinado por el Consejo Nacional Católico para el Ministerio Hispano. ${ }^{38}$ Dicho congreso espera que se continúe el proceso de los encuentros nacionales y su primera reunión ya produjo un documento con líneas de acción, elaboradas por unos 700 líderes hispanos de todo el país. El congreso propone especfficamente reunir a los líderes hispanos, tanto de la sociedad civil como de la Iglesia. Esto supone que, al estilo de los encuentros, los télogos de las bases eclesiales incluyan en el proceso a educadores, legisladores y profesionales en salud. 39 Un segundo congreso esta programado para agosto de 1996, en Chicago.40 La semilla, pues, ha caído en buena tierra y aunque el trabajo y el sacrificio siguen siendo necesarios, el fruto obtenido es abundante.

Preguntémonos, para terminar, cuáles son algunos de los desaflos que esta teología afrontará en los próximos veinticinco años. Tal vez los desaffos pueden ser resumidos de esta manera: cómo construir puentes con otras tradiciones de fe, puentes entre el pueblo y los expertos teólogos, y puentes entre la teologia y

36. Nueve de los diez autores que colaboraron en el proyecto no son latinos y varios de ellos nunca habian escrito antes sobre el asunto. Como estos expertos no latinos, comisionados por la Conferencia Católica de Estados Unidos, están tomando muy en serio los asuntos de los latinos, este trabajo conjunto puede ser un signo de la creciente influencia de la presencia hispana de Estados Unidos y de la perspicacia teologica.

37. Lampe, op. cit.

38. En agosto de 1992, en Los Angeles, California, el encuentro no incluyó reuniones masivas, pero esto puede ser incluido en el futuro. Los diálogos o encuentros actuales entre el pueblo y sus representantes podrían ser muy significativos.

39. National Congress "Roots and Wings": Conclusiones and Action Lines, Los Angeles, California, Consejo Nacional Católico para el Ministerio Hispano, 1992.

40. Véase mi artículo, "De Encuentro a Reconocimiento: The U.S. Hispanic Churd

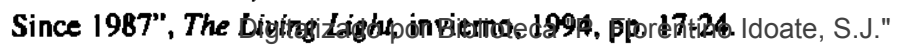

Universidad Centroamericana José Simeón Cañas 
las ciencias sociales.

En 1992 y de nuevo en 1995, el obispo Ricardo Ramírez insistió en que hay que dar prioridad al ecumenismo y un eminente leólogo protestante, Justo González, ha afirmado - reiteradamente- lo mismo.41 No existe todavía un foro para propiciar estc anhclado ecumenismo, aunque la conferencia ecuménica "Aliados en Jerusalén", que tuvo lugar en la Universidad Drew, en 1994. fue ya un buen comienzo. Drew ha sido un líder en el ecumenismo desde la primera reunión ecuménica hispana de 1992.42

A medida que los leólogos hispanos son más aceplados y reconocidos por los profesores de las universidades y las asociaciones profesionales, su inserción dentro de la realidad de los latinos y latinas (quienes a menudo tienen poco que ver con dichos lugares y sectores de influencia) constituirá para ellos un mayor desalío. Sin embargo, si esta teología va a ser la voz del pueblo, entonces la lengua tiene que permanecer junto al cuerpo.

Es difícil tener contacto significativo y personal con algunas comunidades hispanas. Esta es la razón por la que los teólogos y otros líderes deben siempre actualizarse con respecto a los innumerables cambios que afectan a los latinos y latinas en Estados Unidos. Por ejemplo, los ciudadanos de Centromérica exceden ahora en número a los portorriqueños que viven en el continente. ¿No será que la presencia de los centroamericanos en Los Angeles, más la lalta de contacto entre ellos y el liderazgo hispano establecido en esa ciudad, pueden haber exacerbado los disturbios que se dieron allí. La inmigración mexicana ya no es sólo un fenómeno de hombres y la migración interna a menudo pone a los latinos en una situación incómoda con respecto a otros grupos oprimidos.43 Es un reto constante para los líderes entender y acompañar esta situación, que cambia tan rápidamente. La cooperación interdisciplinar entre los teólogos y los sociólogos puede ayudar a enfrentar estos retos.

Esta es la razón por la que la teología de los latinos y latinas en Estados Unidos ha tratado y debe continuar tratando de ser una teología-puente: una teología que hace puentes entre los grupos divididos por diferencias de religión,

41. Voces: Voices from the Hispanic Church, Nashville, 1992, p. 169. Véanse las observaciones del obispo Ramírez en Origins 24 (40) marzo 23, 1995, pp. 660-667, y el reportaje en la edición del 20 de mayo de 1995 del Carholic Trends. Joel N. Martínez adopta una postura semejante desde una perspectiva protestante, en "The People on the Go: The Churh of the Way", Apuntes 15 (2) verano, 1995, pp. 58-7I.

42. El programa de verano hispano reune a seminaristas y a otras personas de varias tradiciones de fe para cursos intensivos, impartidos por profesores latinos. La dimensión ecuménica ha sido uno de sus grandes logros.

43. Véase Jack Miles, "Black Vs. Browns", The Allantic Monshly 270 (4) octubre. 1992. pp. 41-68. Tambien Sleven A. Holmes, en The New York Times 3 (marzo. 1994) A9. 
raza, nacionalidad, sexo y clases. Dicha leología deberá cambiar estos relos en oportunidades; transformar esta tensión en lluvia de esperanza, que caerá sobre estas semillas, sembradas desde hace mucho tiempo.44 La lluvia de oro sobre estas semillas de plata producirá abundante fruto cuando llegue la estación y será suficiente para alimentar a todos los pobres preferidos de Dios.

Ella gritó, "iporque la semilla del mal está implantada dentro de tu alma, ahora mismo! Y ésta es la desgracia - ime escuchas?- la desgracia de nuestro pueblo desde que los españoles llegaron a nuestro suelo: jodio a si mismo! Y debe parar, mi hijito, ¡debe parar ahora mismo! ¡Porque éste es el gran plan de Dios; que el pueblo se alce más allá de sus odios personales, aquí, ahora mismo, en esta nueva tierra donde tanta gente diferente con tanta sangre diferente ha llegado a unirse, y que nosotros reconozcamos que nosotros somos todos hijos de Dios! ¡Cada uno de nosotros!".45

44. Soy consciente que estoy ampliando el concepto de teología de puente o de frontera utilizada por Bañuelas y otros téblogos.

45. El consejo de doña Margarita a su hijo Juan Salvador, de la novela de Víctor Villaseñor, Rain of Gold, Houston, 199I, p. 525. Reconozco mi deuda a Villaseñor por la metáfora utilizada en el título de este artículo. 